\title{
ESTILOS DE ENSEÑANZA UTILIZADOS POR LOS ENTRENADORES DE FÚTBOL BASE EN EL VALLE DEL CAUCA
}

\author{
TEACHING STYLES USED BY BASE \\ FOOTBALL COACHES IN THE CAUCA \\ VALLEY
}

\author{
Germán Darío Isaza Gómez \\ Heriberto González Valencia² \\ Diana Marcela Osorio Roa ${ }^{3}$
}

\section{RESUMEN}

Los estilos de enseñanza se fundan en el conjunto de acciones mediadoras que se adoptan

1 Docente-investigador en Institución Universitaria Escuela Nacional del Deporte. Doctorando en Educación, Universidad de la Salle, Costa Rica; Magíster en Educación, Universidad de Caldas, Colombia; Licenciado en Educación Física y Recreación, Universidad de Caldas, Colombia. Director del Grupo de Investigación Educar 2030. Correo electrónico german.isaza@endeporte.edu.co ORCID https:// orcid.org/0000-0001-8475-9994 Cali, Colombia.

2 Docente-investigador en Institución Universitaria Escuela Nacional del Deporte. Doctorando en Investigación en Humanidades Artes y Educación de la Universidad Castilla La Mancha, España. Magister en Educación, y, Especialista en Educación y Licenciatura en Lengua Extranjera. Universidad Santiago de Cali. Miembro del Grupo de Investigación Educar 2030. Correo electrónico: heriberto.gonzalez@endeporte.edu.co Orcid: https://org/0000-0001-91032152 Cali, Colombia.

3 Docente-investigador Universidad Javeriana Cali. Magister en Ciencias Biomédicas, Universidad del Valle. Fisioterapeuta, Institución Universitaria Escuela Nacional del Deporte. Miembro del Grupo de Investigación en Ciencias Básicas y Clínicas para la Salud. Correo diana.osorio@javerianacali.edu.co Orcid: https://org/0000-0002-6405-699X Cali, Colombia. en las relaciones didácticas que implementa el entrenador para la organización de sus actividades de entrenamiento y las relaciones que establece en el proceso de enseñanzaaprendizaje. El objetivo del estudio permitió reconocer la formación profesional y los estilos de enseñanza de los entrenadores de fútbol base del Valle del Cauca. La metodología siguió la lógica descriptiva, la muestra de tipo no probabilístico por conveniencia y carácter voluntario estuvo constituida por 277 entrenadores con una edad promedio de $38 \pm 12$,2 años, y una experiencia promedio en el ejercicio de su profesión de 12,3 $\pm 9,1$ años, los instrumentos utilizados fueron la encuesta sociodemográfica y el instrumento de estilos de enseñanza de teorías implícitas. Los resultados evidencian que los entrenadores empíricos con una experiencia novel presentan una tendencia hacia la 
utilización de estilos de enseñanza tradicional y técnica, mientras que los entrenadores con titulación profesional tienen una tendencia hacia estilos activos e interpretativos. En conclusión, la formación académica es fundamental en los procesos pedagógicos en el campo del deporte. Los entrenadores empíricos y con titulación profesional con poca experiencia no demuestran prácticas de enseñanza enfocadas hacia el estilo crítico; sin embargo, se evidencia un desplazamiento de estilos de enseñanza tradicional hacia estilos más comprensivos a partir de la experiencia en el ejercicio de su profesión.

\section{PALABRAS CLAVE}

Estilos de enseñanza; formación académica; fútbol base, entrenadores, experiencia profesional.

\section{ABSTRACT}

Teaching styles are based on the set of mediating actions that are adopted in the didactic relationships that the coach implements, the organization of his training activities and the relationships that he establishes in the teaching-learning process. The objective of the study allowed us to recognize the professional training and teaching styles of the youth soccer coaches of Valle del Cauca. The methodology followed the descriptive logic, the non-probabilistic sample for convenience and voluntary character consisted of 277 trainers with an average age of $38 \pm 12.2$ years, and an average experience in the exercise of their profession of $12.3 \pm 9.1$ years, the instruments used were the sociodemographic survey and the implicit theory teaching styles instrument. The results show that empirical trainers with novice experience show a tendency towards the use of traditional and technical teaching styles, while trainers with professional qualifications have a tendency towards active and interpretive styles. In conclusion, academic training is essential in pedagogical processes in the field of sport. Vocational and empirical trainers with little experience do not demonstrate critical style focused teaching practices; However, there is evidence of a shift from traditional teaching styles to more comprehensive styles based on the experience in the exercise of their profession.

\section{KEYWORDS}

Teaching styles; academic training; grassroots football, coaches, professional experience.

\section{INTRODUCCIÓN}

El presente resultado de investigación pertenece al proyecto titulado "Determinación de las mediaciones educativas de los entrenadores deportivos del Valle del Cauca", llevado a cabo por el grupo de investigación Educar 2030 de la Institución Universitaria Escuela Nacional del Deporte. El proyecto pretende generar una profunda reflexión sobre los procesos pedagógicos abordados en el campo del deporte, donde los estilos de enseñanza se erigen como el escenario mediador donde los entrenadores trasmiten y facilitan los aprendizajes a sus deportistas.

Está corriente pedagógica ha generado un interés particular en investigadores educativos y deportivos por reconocer como se están mediando los procesos pedagógicos no solo en el campo del deporte (Isaza-Gómez, et al., 2021; Zuluaga, Isaza-Gómez y Osorio-Roa, 2021; Rodríguez y Quilindo 2021; Fuentes, Ries y Rodríguez, 2020; Bernardino, et al., 2017; Delgado, 2015; Ambris, 2013) sino en la educación en general, (Arellano, 2018; Patiño, 2012).

Los procesos de mediación pedagógica en el entrenamiento deportivo constituyen el elemento esencial en el desarrollo de la enseñanza de los deportes, en especial el fútbol, el cual es practicado por miles de niños y niñas en el planeta desde edades tempranas 
y aun no existe un acuerdo específico entre los entrenadores sobre cuales métodos o estilos de enseñanza utilizar en los procesos de formación deportiva, los cuales se han centrado en procesos enfocados en los métodos analíticos, tradicionales y reproductivos de enseñanza, (Rodríguez y Quilindo, 2021; Pérez-Muñoz, et al., 2021; Gómez, 2020) así las cosas, es necesario ampliar la mirada de los procesos de enseñanza, a estilos más comprensivos y participativos, donde el centro del proceso de aprendizaje sean los deportistas (Guzmán y Villalobos, 2019; Patiño, 2012; Isidori y Fraile, 2011; Lyle, 2007).

Cada entrenador utiliza los estilos de enseñanza que cree más convenientes para formar a los jugadores que tiene a su cargo. Sin embargo, la elección de estos estilos se ve muchas veces condicionada por el club en el que trabaja o por las características de sus jugadores, así como por otros aspectos como las instalaciones o los recursos humanos y materiales de los que dispone, García, 2021; Pérez-Muñoz, et al., 2020; Rodríguez, 2016). Pero trabajar en el fútbol base también exige a los entrenadores estar en un proceso de formación continuo, donde en muchas ocasiones el entrenador tiene que conocer el desempeño de la labor de otros profesionales para poder valorar y mejorar su propia actuación, con el fin de aumentar la calidad de sus sesiones de entrenamientos $y$, en consecuencia, la de sus jugadores. Si bien los partidos de competición son un gran escenario para el aprendizaje, es en las sesiones de entrenamiento donde el entrenador propone los ejercicios y las metodologías que ellos creen más adecuadas para formar a sus jugadores a nivel técnico-táctico, en valores y formación ciudadanía, (Hernández y Gracia, 2018; Gómez, Cruz y Moya, 2018; Gómez, 2016; Monjas, Ponce y Gea, 2015; Cerezo, 2019; Nuviala, León y Fernández, 2007).
También se puede definir que el entrenamiento deportivo es un proceso conducido de forma planificada, en virtud del cual han de desarrollarse unos cambios en el estado de rendimiento motriz, por otra parte los entrenadores deben capacitarse con respecto a las nuevas tendencias del entrenamiento deportivo infantil para evitar cometer errores debido que a los niños se les aplican procesos como si fueran adultos, es por ello que muchos de los deportistas han abandonado estas prácticas a tempranas edades (Maestre, et al., 2018; Pedraza, et al., 2015; Jiménez, Rodríguez y Castillo, 2001).

Los entrenadores se basan mucho en el desarrollo de capacidades físicas, técnicas y el juego competitivo del fútbol, descuidando los procesos pedagógicos, entre ellos los estilos de enseñanza que deberían orientar correctamente a los deportistas en el entrenamiento para un óptimo aprendizaje de los fundamentos básicos del fútbol base. La enseñanza del fútbol es compleja, los entrenadores deben reconocer las características individuales de cada deportista, sus habilidades y capacidades, a fin de potenciarlas en sus trabajos colectivos, fomentar procesos de descubrimiento guiado a través de la comunicación y participación colectiva entre la bina entrenador deportista que promueva espacios que estimule la creación y la resolución de problemas a través de la toma de decisiones.

Los estudios de Isaza-Gómez, et al., (2021); Zuluaga, Isaza-Gómez y Osorio Roa (2021); Rodríguez y Quilindo (2021) han realizado un esfuerzo por reconocer los estilos de enseñanza utilizados en el campo del deporte en el Valle del Cauca, reconociendo en este campo del saber un escenario potente para fomentar procesos deportivos más comprensivos y críticos a fin de desplazar las formas más instituidas en el campo del deporte desde la enseñanza tradicional y analítica. 
Reconocer los procesos relacionados con los estilos de enseñanza como campo de conocimiento, necesariamente remite a los trabajos de Mosston y Ashworth (1986), Ibañez y Medina (1999), Delgado (1991), Onofre (1998), Camacho (2003), Valencia (2005) y Zurita (2016) quienes realizan un esfuerzo académico por reconocer los estilos de enseñanza utilizados en los procesos de educación física y los deportes.

Los estilos de enseñanza se logran consolidar en cuatro grandes categorías, estilos tradicionales, Ios que promueven la participación del deportista/ estudiante, los que estimulan el desarrollo cognitivo y el desarrollo de la creatividad. En este sentido los estilos de enseñanza se configuran en un escenario donde se puede visibilizar las teorías generales de la pedagogía aplicada al deporte.

Los estudios de Delgado-Noguera (1991) propone algunos estilos de enseñanza en la Educación Física: propuesta para una reforma de la enseñanza, donde los define como:

Definimos el estilo de enseñanza como modo o forma que adoptan las relaciones didácticas entre los elementos personales del proceso de enseñanza-aprendizaje tanto a nivel técnico y comunicativo, como a nivel de organización del grupo de la clase y de sus relaciones afectivas en función de las decisiones que tome el profesor" (p.18).

En este sentido, los estilos se atribuyen a una serie de características propias del entrenador, quien condiciona su estilo a una serie de categorías tales como la experiencia deportiva previa a su rol como entrenador, el nivel de estudios alcanzado, su experiencia en el ejercicio de su profesión y comprensión de los alcances pedagógicos aplicados en cada proceso formativo.
Camacho (2003) resalta la importancia de que cada maestro sea el autor de su propio estilo de enseñanza, sin rechazar los demás estilos, sino por el contrario acoplando lo mejor de cada uno en consecución de sus propios objetivos como formador. Es importante que cada maestro construya su estilo de enseñanza entendiendo que son "...la forma peculiar que tiene cada profesor de elaborar el programa, aplicar el método, organizar la clase y relacionarse con los alumnos, es decir, el modo de llevar la clase." (p.27).

Por su parte, Marrero (1993) en su trabajo articula un instrumento enfocado en evaluar lo que él denomina las teorías implícitas, dicha teoría se fundamenta en los estilos de enseñanza personales que cada formador ha implementado a lo largo de su desarrollo, gracias a lo que se le ha transmitido en su formación, así como toda la teoría que el mismo utilizó como base para desarrollar su conocimiento, los cuales los divide en cinco estilos de enseñanza: Tradicional, técnico, interpretativo, activo y critico argumentando que como formadores siempre se utiliza al menos un porcentaje de cada uno de los estilos en los procesos de formación deportiva.

Así las cosas, el estudio pretende reconocer la formación profesional y los estilos de enseñanza utilizados por los entrenadores deportivos, es necesario ampliar el mapeo pedagógico de los formadores deportivos en el Valle del Cauca, ya que algunos entrenadores reconocen la importancia de los procesos pedagógicos en el campo del deporte, a diferencia de otros que trabajan de manera empírica y no tienen presente los procesos pedagógicos es sus actividades de entrenamiento (Monjas, et al., 2015; González, 2012; Castejón, 2008; Nuviala, León, Gálvez y Fernández, 2007). 


\section{METODOLOGÍA}

El estudio siguió la lógica del enfoque cuantitativo, donde se oriento hacia el estudio descriptivo, el cual permitió determinar los estilos de enseñanza utilizados por un grupo de entrenadores de fútbol base del Valle del Cauca. La población estuvo constituida por todos los entrenadores de fútbol base del departamento; la muestra de tipo no probabilístico por conveniencia y carácter voluntario estuvo constituida por 277 entrenadores. Los criterios de inclusión fueron: Ser entrenadores en el departamento del Valle del Cauca, ser formador en una de las categorías de fútbol base y aceptar voluntariamente participar en el estudio. El estudio estuvo aprobado por el comité de ética de la Institución Universitaria Escuela Nacional del Deporte.

Los instrumentos utilizados fueron la encuesta sociodemográfica y el instrumento de estilos de enseñanza "teorías implícitas". La encuesta sociodemográfica, fue construida y adaptada a las condiciones sociodemográficas de los entrenadores del Valle del Cauca, siguiendo los estudios de García Ferrando (2002), donde se adaptó el cuestionario de situación profesional de los recursos humanos de actividad física y deporte (Campos-Izquierdo, 2003), ya validado en estudios sobre recursos humanos del deporte (Campos- Izquierdo, 2007; Gallardo y CamposIzquierdo, 2010).

El instrumento sobre los estilos de enseñanza fue validado por Marrero (1993) a través del cuestionario de teorías implícitas relacionado con los estilos de enseñanza, conceptualmente son teorías pedagógicas personales construidas sobre la base de conocimientos pedagógicos históricamente elaborados y trasmitidos a través de la formación y en la práctica pedagógica y desdeel punto de vista operacional es la diferencia entre las sumas finales de los ítems asociados a cada enfoque teórico (Tradicional, técnico, interpretativo, activo y critico). Es un instrumento validado con diversos tipos de población ya que ha sido utilizado en investigaciones anteriores Judukis (2007) y utilizado en el ámbito deportivo por Ponce (2006). Este instrumento consta de 33 ítems valorados por una escala Likert de cuatro opciones (1= Muy en Desacuerdo; 2= En Desacuerdo; 3=De Acuerdo y 4= Muy de Acuerdo); de esta forma, a los entrenadores se les muestran una serie de afirmaciones y se le indica que marquen su valoración eligiendo una de las cuatro opciones propuestas en nuestra escala, indicando nuevamente que tiene una dirección negativa o desfavorable (implicaría una menor puntuación) o negativa así como positiva o favorable (supondría una mayor puntuación).

El análisis de la información se realizó a través del programa de office Excel, por medio de estadística descriptiva básica, donde se logró evidenciar los diferentes estilos de enseñanza.

\section{RESULTADOS}

El estudio se realizó con 277 entrenadores de fútbol de categorías formativas del departamento del Valle del Cauca, Colombia, donde el $95 \%$ pertenece al sexo masculino y el $5 \%$ al femenino, con una edad promedio de 38 \pm 12 ,2 años, y una experiencia promedio en el ejercicio de su profesión de 12,3 \pm 9,1 años. En la siguiente tabla, se presenta la experiencia de los entrenadores: 
Tabla 1. Experiencia de los entrenadores.

\begin{tabular}{|c|c|c|c|}
\hline Rango & Años & $\mathrm{n}$ & $\%$ \\
\hline Novato & 0 a 5 & 118 & 42,6 \\
\hline Media & 6 a 10 & 67 & 24,2 \\
\hline Expertos & 11 en adelante & 92 & 33,2 \\
\hline Total & & 277 & $100 \%$ \\
\hline
\end{tabular}

Fuente: Elaboración propia.

Como se evidencia en la tabla anterior, el $43 \%$ de los entrenadores evidencia tener una experiencia inicial como entrenador de fútbol, y solo el $33 \%$ de los entrenadores evidencia una alta experiencia en los procesos de formación deportiva. El nivel de formación académica de los entrenadores de fútbol (Tabla 2) evidencia que el $70 \%$ de los encuestados tiene un nivel académico superior al de bachillerato.

Tabla 2. Nivel de formación de los entrenadores de fútbol base.

\begin{tabular}{|l|c|c|}
\hline Nivel de formación & $\mathrm{n}$ & $\%$ \\
\hline Bachillerato & 82 & 29,6 \\
\hline Tecnológico & 41 & 14,8 \\
\hline Pregrado & 152 & 54,9 \\
\hline Posgrado & 2 & 0,7 \\
\hline Total & 277 & $100 \%$ \\
\hline
\end{tabular}

Fuente: Elaboración propia.

De las 195 titulaciones se identificó el 70\% tiene titulación en áreas afines a su profesión como entrenador, ya sea con títulos tecnológicos en el campo del deporte, profesional en deporte o como licenciado en educación física y recreación. De los entrenadores encuestados del departamento del Valle del Cauca, el $41 \%$ de ellos realizan cursos periódicos de actualización, donde se destacan: A nivel local 14\% realizaron cursos de actualización en semilleros e instituciones oficiales, tales como: la Universidad del Valle, el Servicio Nacional de Aprendizaje (SENA) y la institución de la Escuela Nacional del Deporte. A nivel regional $25 \%$ se realizan cursos en la liga de fútbol vallecaucana donde se destaca el curso regional de entrenadores auspiciado por INDERVALLE. A nivel nacional el $11 \%$ ha obtenido certificación de licencias A, B y/o $C$ otorgadas por la Federación Colombiana de Fútbol (FCF) y Coldeportes, las cuales se cursan en diferentes universidades del país. A nivel internacional el $5 \%$ realizaron algunos de los siguientes cursos: Licencias UEFA, AFA y FIFA.

\section{ESTILOSDEENSEÑANZAPREDOMINANTES.}

Los estilos de enseñanza (Tabla 3) demuestran la tendencia en las mediaciones pedagógicas implementadas por los entrenadores durante las sesiones de entrenamiento, donde se logra evidenciar una tendencia hacia la utilización el estilo de enseñanza activo (44\%), mientras que el menos utilizado es el estilo crítico (4\%).

Tabla 3. Estilos de enseñanza utilizados por los entrenadores.

\begin{tabular}{lcc}
\hline Estilo & $\mathbf{n}$ & $\%$ \\
Tradicional & 34 & $12 \%$ \\
Técnico & 38 & $14 \%$ \\
Interpretativo & 70 & $25 \%$ \\
Activo & 123 & $44 \%$ \\
Crítico & 12 & $4 \%$ \\
\hline Total & 277 & $100 \%$ \\
\hline
\end{tabular}

Fuente: Elaboración propia.

Con el propósito de analizar los estilos de enseñanza con un cuidado especial, se distribuyeron los entrenadores en empíricos y los que poseen titulación profesional (Gráfica 1) donde se logra evidenciar que los estilos 
con mayor preponderancia en los entrenadores con titulación profesional son los activos e interpretativos, mientras que los empíricos emplean en estilos activos y tradicionales. Se observa en una menor proporción utilización de estilos críticos en sus procesos de enseñanza.

Gráfica 1. Estilos de enseñanza entrenadores con titulación profesional y empíricos.

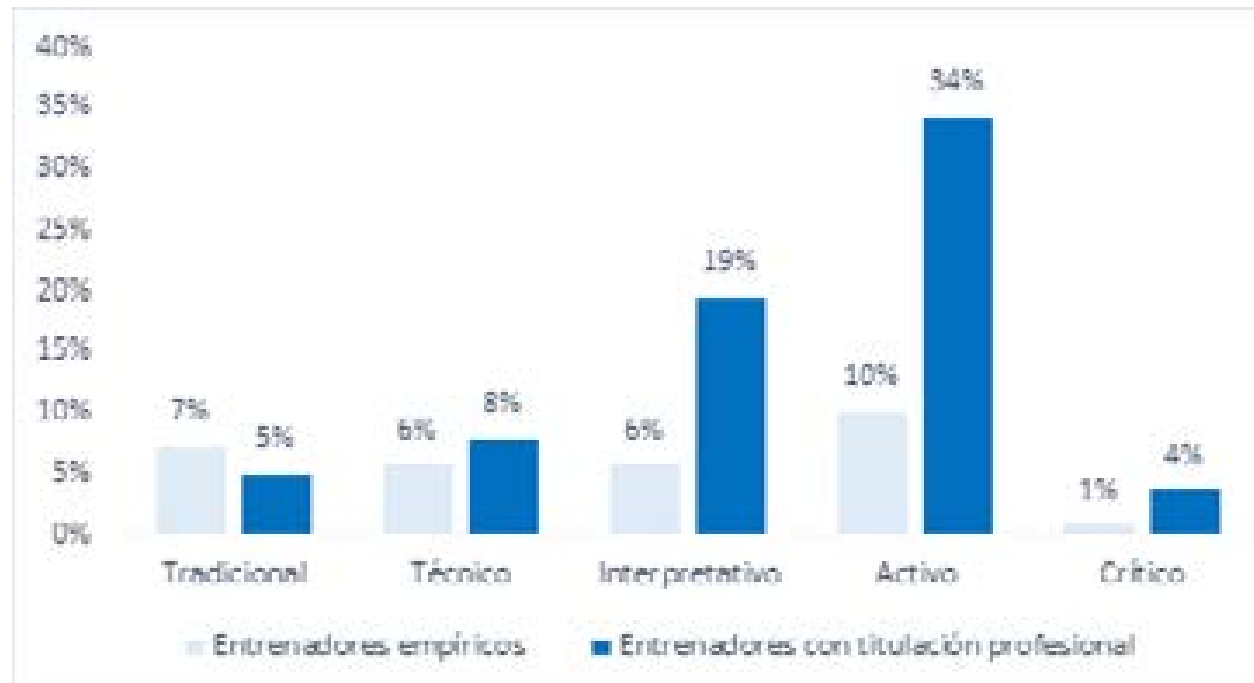

Fuente: Elaboración propia.

Es claro que las formas de enseñar se transforman a través del tiempo, en este estudio para una mejor comprensión de los resultados se logró dividir la experiencia en el ejercicio de la profesión en tres categorías: Entrenadores novatos con una experiencia inferior a cinco años; el entrenador con experiencia media con un tiempo dedicado a su profesión entre cinco y diez años; finalmente, el entrenador experto con más de diez años de experiencia en el medio. Allí se logra develar datos significativos relacionados con los estilos de enseñanza, donde los entrenadores novatos empíricos utilizan de manera significativa el estilo tradicional, mientras que los entrenadores en ese mismo rango de experiencia utilizan estilos activos. Solo después de mudar a una experiencia superior a los 10 años los entrenadores empíricos logran transformar sus estilos de enseñanza a unos más activos. 
Gráfica 2. Estilos de enseñanza entrenadores con titulación profesional según su experiencia.

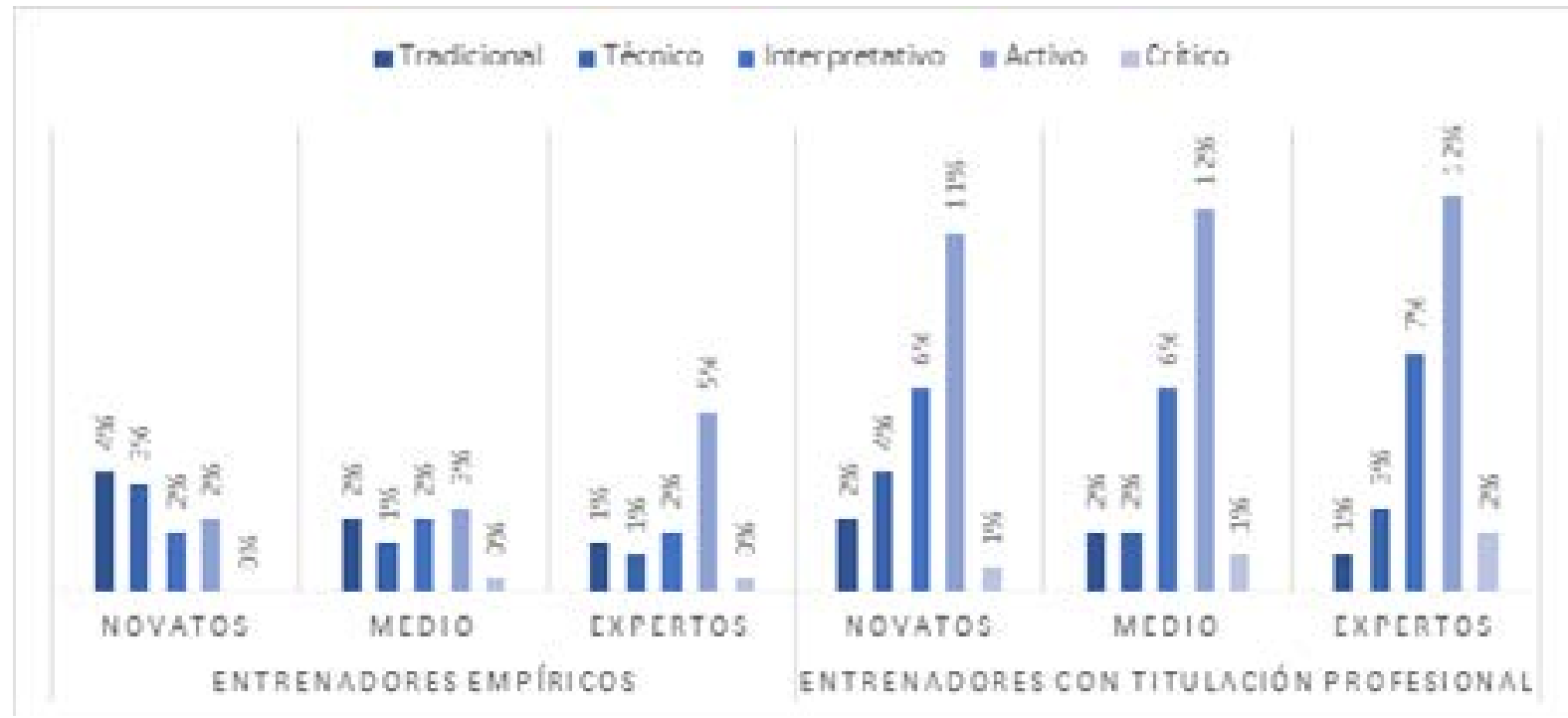

Fuente: Elaboración propia.

Los entrenadores deportivos del Valle del Cauca se caracterizan por haber practicado deporte en su juventud, el $38 \%$ lo realizó a nivel profesional, mientras que el $62 \%$ a nivel amateur. Igualmente el estudio determina que ambos tipos de entrenadores con experiencia deportiva amateur y profesional tienen una mayor tendencia a la utilización de estilos activos, mientras que los entrenadores que los que menos utilizan son los estilos críticos.

Gráfica 3. Estilos de enseñanza entrenadores con experiencia deportiva profesional y amateur.

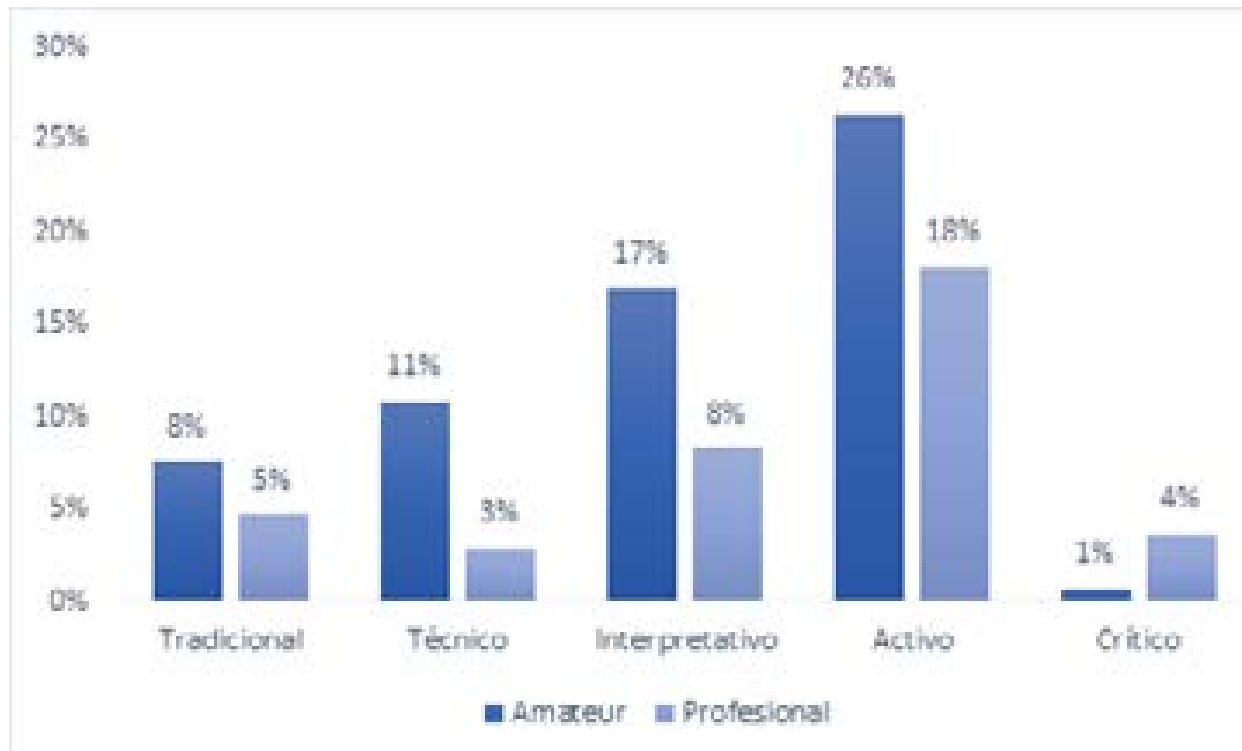

Fuente: Elaboración propia. 


\section{DISCUSIÓN}

El objetivo de este estudio fue analizar la formación académica y estilos de enseñanza de los entrenadores de fútbol base del Valle del Cauca, los resultados evidencian una mayoría de los entrenadores de fútbol en el Valle del Cauca pertenecientes al sexo masculino, mientras $5 \%$ son del sexo opuesto, resultados que concuerdan con los de Isaza, et al., (2021) donde se evidenció que el $93 \%$ de los entrenadores de fútbol corresponde a sexo masculino, igualmente, se encontró un marcado desequilibrio en la investigación realizada por Ayala-Zuluaga, Aguirre-Loaiza y RamosBermúdez (2015), donde el 90\% corresponde a entrenadores hombres y el $10 \%$ a entrenadoras mujeres, estudios como los de Robles, Fuentes, Rodríguez y López (2011) refleja que una gran parte de los entrenadores de educación física y diversos campos del deporte son de sexo masculino $99 \%$ y solo el $1 \%$ corresponde al sexo femenino; es evidente como en asuntos relacionados con las mediaciones pedagógicas en el campo del deporte y en especial del fútbol existe una tendencia hacia los entrenadores masculinos con relación a las entrenadoras femeninas.

Los resultados evidenciaron que el $30 \%$ de los entrenadores en el Valle del Cauca son empíricos, datos que concuerdan con los estudios de Zuluaga, Isaza y Osorio, (2021) quienes encontraron que al menos $27 \%$ de los entrenadores carecen de estudios de pregrado en el campo del deporte; asimismo, Manrique, Gea y Garzón (2013) encontraron que el $41 \%$ de los entrenadores no presentan titulaciones en el campo de las ciencias de la actividad física y el deporte. Los estudios de Lledó, Martínez y Huertas (2014) demostraron que solo el $60 \%$ de los entrenadores de categorías formativas de clubes de primera división de la comunidad valenciana, tienen una titulación universitaria. Así las cosas, no solo en el Valle del Cauca, sino en diferentes escenarios latinoamericanos e inclusive europeos, es necesario profesionalizar el campo del deporte y áreas afines, donde a través de políticas públicas en deporte se pueda exigir a las personas que desean ejercer en el campo del deporte al menos titulación técnica profesional para poder desempeñar su profesión, está comprobado que este tipo de entrenadores evidencian unas prácticas de enseñanza mediadas por el ensayo-error con sus deportistas.

La profesionalización del ejercicio del entrenador es fundamental para el desarrollo del país en términos deportivos, los resultados evidenciaron que cerca del $70 \%$ tienen estudios profesionales, y solo el $1 \%$ presentan titulación de posgrado, así las cosas se evidencia que en estas categorías formativas no es suficiente ni necesario alta especialidad académica, sin embargo, los estudios de Ayala, Aguirre y Ramos (2015) determinan que los entrenadores suramericanos de altos logros tienen una alta cualificación a nivel profesional, inclusive con estudios especializados en el campo del deporte, en este sentido, la acción del entrenador es fundamental para una afrontar los retos permanentes que emergen en el campo del entrenamiento, asegurando no solo procesos pedagógicos acordes al desarrollo de los niños, niñas y jóvenes, sino el desarrollo de las habilidades y capacidades motrices a través de la aplicación de las teorías y metodologías de las ciencias aplicadas al deporte, en este orden de ideas, "los entrenadores mejor formados serán aquellos que podrán afrontar con mayores garantías sus funciones como entrenador" (Feu, et al., 2010, p. 636).

Los estilos de enseñanza que emplean los entrenadores tienen una tendencia hacia el modelo activo, sin embargo al momento de validar estos resultados, se evidencia que los estilos varían dependiendo del nivel de estudios y la experiencia de los entrenadores en el ejercicio de su profesión, por lo tanto se logra evidenciar 
que los entrenadores empíricos, novatos y con una experiencia media tienen una tendencia a la utilización de estilos de enseñanza tradicional, mientras que los entrenadores con titulación académica tienen una tendencia hacia estilos de enseñanza más participativos, estudios que concuerdan con los resultados de Rodríguez y Quilindo (2021) quienes afirman, "Se considera que existe una concordancia entre el método y el estilo de enseñanza tradicional, dado a que este método se utiliza la instrucción directa para la enseñanza de la técnica y el profesor adquiere un protagonismo en este proceso" (p.46).

Por otro lado, se reconoce un desplazamiento de los estilos de enseñanza tradicionales hacia estilos más activos en los entrenadores empíricos, ello da pistas que con la experiencia se logra mudar de estilos, donde empieza a primar estilos de enseñanza más activos, tal como lo cita González, 2012, quien considera "los entrenadores empíricos que fomentan el estilo activo es debido a la experiencia que estos han adquirido a lo largo de su profesión, el conocimiento resulta de la combinación de captar y transformar la experiencia" (p. 3).

La experiencia en el ejercicio de la profesión de los entrenadores se convierte en un escenario de aprendizaje, es fundamental para afinar los procesos de enseñanza, no es lo mismo para un entrenador que inicia en sus primeros pinos laborales, a quien lleva más de 10 años y hasta más en el campo deportivo; la misma experiencia es un escenario para el aprendizaje genera en los formadores una serie de habilidades para gestionar y recrear las sesiones de entrenamiento.

Los procesos de enseñanza se transforman con el paso de los años, García et al., (2017), concuerda que "con el paso de los años el entrenador se va alejando cada vez más de este tipo de estilos que no implican cognitivamente al alumno" (p. 20), es decir, se enfoca en estilos más participativos y se aleja de los estilos tradicionales enfocados en la asignación de tareas, el mando directo y procesos analíticos de enseñanza. En este sentido, es necesario promover escenarios contundentes de formación permanente de los entrenadores en el campo del deporte, ya que los entrenadores con mayor experiencia y titulación tienen una tendencia hacia la utilización de estilos de enseñanza más participativos, donde se estimula cognitivamente a los deportistas. La alta titulación y experiencia en los entrenadores les permite una visión más comprensiva de los procesos de entrenamiento, sin embargo, en el campo deportivo falta promover escenarios de aprendizaje crítico en los deportistas, producto de los recursos limitados que tienen los entrenadores a nivel pedagógico.

En este sentido, los entrenadores noveles y entrenadores empíricos tienen como fin principal ganar a toda costa, olvidando los principios pedagógicos del juego en esta edad los cuales deben ir enfocados hacia el goce y el disfrute. Esto acontece con frecuencia en los diferentes torneos de fútbol base, donde prima el buen nombre y el status del entrenador por encima del proceso deportivo de los niños; quienes están interesados solo en ganar a toda costa, estudios que concuerdan con los de Robles et al., (2011) quienes coinciden que "muchos entrenadores del fútbol base usan a sus jóvenes jugadores como un medio de trepar en la escalera social y profesional, dando prioridad al ganar, más que al desarrollo del jugador" ( $p$. 24). Ganar es importante, sin embargo en esta fase del desarrollo deportivo es necesario que el pequeño futbolista tome decisiones, resuelva problemas y tenga la capacidad de crear diferentes jugadas y situaciones de juego en ambientes de confianza y seguridad.

Es común ver en los diferentes campos de juego entrenadores gritando y dando instrucciones continuamente a sus jugadores, "pasa la pelota" "suben" "bajan" "achiquen" Agranden" "toque, 
toque" condicionando las acciones de los jugadores y limitando constantemente la toma de decisiones y la resolución de problemas, los cuales afectan el desarrollo armónico de los pequeños futbolistas, de allí la importancia de promover escenarios más dinámicos en los diferentes torneos y festivales infantiles, donde se les permita a los niños ser conscientes de sus decisiones dentro del terreno de juego, Huesca (2017) indica que el jugador del fútbol base es el responsable de la toma de decisiones, existen diferentes respuestas y el jugador indaga para lograr descubrir cuál de ellas es la más apropiada y cómo conseguirla. De este modo, los entrenadores y formadores se desempeñan como guías en dicho aprendizaje, motivando en el niño el descubrimiento de sus capacidades y dejando a un lado el estilo donde el entrenador fuese quien proporciona el conocimiento y los deportistas se limitarán a desarrollar o seguir las indicaciones.

El estilo de enseñanza activo es el camino para fomentar estilos con mayor participación en los pequeños jugadores, es necesario romper con los esquemas tradicionalistas de enseñanza y promover estilos más cognitivos y críticos, donde los deportistas puedan ser el centro del desarrollo deportivo a partir de la toma de decisiones y la resolución de problemas, los cuales son fundamentales en las siguientes etapas de formación deportiva.

En este orden de ideas, es importante resaltar la importancia de la implementación constante de los estilos activos e interpretativos; sin embargo, es necesario fomentar procesos de enseñanza basados en estilos críticos, los cuales son fundamentales en el desarrollo del fútbol moderno, donde los deportistas deben evaluar rápidamente la ubicación de la pelota, sus compañeros y oponentes, e incluso la de ellos mismos, antes de tomar una decisión basada en sus habilidades, dicho de otra manera Schumacher, et al., (2018) menciona: "Con el objetivo de conseguir un rendimiento excelente en este campo, es clave en dicho proceso los siguientes aspectos: percibir la situación; procesar los estímulos; tomar la decisión correcta lo más rápido posible y ejecutar la acción en el momento oportuno" (p. 3).

Así las cosas, promover estilos de enseñanza más críticos, interpretativos y activos contribuyen al desarrollo cognitivo de los pequeños jugadores, quienes aprenden a jugar en medio de características complejas, preparándolos constantemente a responder a múltiples situaciones que se pueden presentar en el terreno de juego, Isidori y Fraile (2011) coinciden en que estas perspectivas pedagógicas críticas, consigan una mayor comprensión de su labor docente, reflexionando sobre su actuación en la práctica y promoviendo con ello el cambio social que trata de alejarse de las visiones tradicionales hegemónicas establecidas en el ámbito de estudio de las ciencias del deporte (p.144)

\section{REFERENCIAS BIBLIOGRÁFICAS}

Ambris, J. C. (2013). Estilos de enseñanzaaprendizaje en relación con la satisfacción y ansiedad que provoca el fútbol en edades tempranas en ciudad del Carmen (México). Tesis Doctoral.

Arellano, P. R. (2018). Planteamiento de los estilos de enseñanza desde un enfoque cognitivo-constructivista. Tendencias pedagógicas, (31), 47-68.

Ayala-Zuluaga, C., Aguirre Loaiza, H., y Ramos Bermúdez, S. (2015). Formación académica y experiencia deportiva de los entrenadores suramericanos (pp. 368378).

Bernardino, J., García, F., Martínez, A. y Javier, V., (2017). Los estilos de enseñanza en los entrenadores de fútbol. Murcia España, pp.15 - 21. 
Camacho Coy, H. (2003). Pedagogía y didáctica de la educación física (No. 796.07 C172p). Editorial Kinesis.

Campos-Izquierdo, A. (2003). La organización de la actividad física y del deporte en las instalaciones deportivas de la Comarca Burgalesa de la Ribera del Duero respecto a los profesionales de la actividad física y del deporte. En Universidad de Granada (eds), II Congreso Mundial de Ciencias de la actividad física y del deporte. Granada: Universidad de Granada.

Campos-Izquierdo, A. (2007). Los profesionales de la actividad física y deporte como elemento de garantía y calidad de los servicios. Cultura, Ciencia y Deporte, 7 , 51- 57.

Castejón, F. (2008). Deporte escolar y competición. En I. Hernández y $F$. Martínez (Coords.). El deporte escolar en la sociedad contemporánea. (pp. 159177). Almería: Universidad de Almería.

Cerezo, J., (2019). Diseño de una propuesta metodológica para el desarrollo de las competencias ciudadanas a través del fútbol sala, en escolares entre 11-13 años. Repositorio Digital San Buenaventura, pp.94-126.

Delgado, M., (2015). Los estilos de enseñanza de la educación física y el deporte a través de 40 años de vida profesional. Granada - España, pp.240 - 247.

Feu, S., Ibáñez, S. J. y Gozalo, M. (2010). La formación inicial de los entrenadores de balonmano para la enseñanza del deporte en la edad escolar. Cultura, Ciencia y Deporte, 5(14), 109- 117.
Fuentes, W. R. C., Ries, F., \& Rodríguez, M. C. (2020). Estilos de aprendizaje y ambiente de aula: situaciones que anteceden a la innovación pedagógica en estudiantes de deporte. Retos: nuevas tendencias en educación física, deporte y recreación, (38), 213-221.

Gallardo, J., \& Campos-Izquierdo, A. (2011). Situación profesional de los recursos humanos de la actividad física y del deporte en el municipio de Coslada. Revista Internacional de Medicina $y$ Ciencias de la Actividad Física y del deporte, 43, 440-454.

García Ceberino, J. M. (2021). Estudio de las metodologías para la enseñanza del fútbol en la edad escolar. Universidad de Extremadura. Tesis doctoral.

García Ferrando, M. (2002). La encuesta. En F. Alvira, M. García Ferrando \& J. Ibáñez (comps.), El análisis de la realidad social. Métodos y técnicas de investigación (pp. 167-202). Madrid: Alianza Editorial.

García Angulo, F.J.; Valero Valenzuela, A.; Sánchez-Alcaraz Martínez, B.J. (2017). Estilos de enseñanza en los entrenadores de fútbol. Trances, 9(1):15-22.

Gómez, G. D. I. (2016). Proyecto: Misión ENDeporte. RedPensar, 5(2), 1-16.

Gómez, G. D. I., Cruz, R. R., \& Moya, J. L. C. (2019). Experiencias pedagógicas significativas de transformación social y construcción de paz a través de la formación deportiva en la ciudad de Santiago de Cali, Colombia/Endeporte Mission: Significant Pedagogical Experiences of Social Transformation and Peacebuilding through Sports Training in the City of Santiago de Cali, Colombia. Revista Internacional de Educación y Aprendizaje, 6(4), 201-207. 
Gómez Medina, J. S. (2020). Comparación del método analítico y el método globalizado en el fútbol base en la categoría Benjamín (Doctoral dissertation, Corporación Universitaria Minuto de Dios).

González, M. (2012). Evaluación del funcionamiento del primer curso de implantación de un Programa Integral de Deporte Escolar en Educación Primaria en el municipio de Segovia (Tesis Doctoral). Segovia: Universidad de Valladolid.

Guzmán, E. H. y Villalobos, L. J. (2019). Influencia del modelo didáctico-crítico, en el desempeño de los docentes en formación de Licenciatura en Educación Básica con énfasis en Educación Física, Recreación y Deportes de la Universidad Libre un estilo diferente de enseñanza. Recuperado de: https://hdl.handle. net/10901/18017.

Hernández Merchán, A. C., y Gracia Díaz, Álvaro J. (2018). Entrenamiento pedagógico en habilidades motrices, capacidades condicionales técnico tácticas y formación en valores dentro y fuera de la cancha como proceso de formación en el Club Porto F.C. Revista Digital: Actividad Física y Deporte, 2(1). pp. 50-64.

Huesca, J. (2017). Estilos de enseñanza en fútbol base. Recuperado de https:// mundoentrenamiento.com/estilosensenanza-fútbol-base

Ibáñez Godoy, S. J., \& Medina Casaubón, J. (1999). Relaciones entre la formación del entrenador deportivo y la formación del profesor de Educación Física. Apunts. Educació física i esports.
Isaza-Gómez G, D; González-Hernández, E; González-Valencia, H; Villota-Enriques, J, A; Torres-Ortiz, J, D; Grajales-Hurtado, S. (2021). Mediaciones pedagógicas de un grupo de formadores de fútbol de la ciudad de Cali. En: Isaza-Gómez, G D; Osorio-Roa, D M; \& González-Valencia, H (ed científicos). 2021. Mediaciones pedagógicas y educativas en el campo del deporte. Cali, Colombia. Editorial ASACOL Services, LLC.

Isidori, E., \& Fraile, A. (2011). La pedagogía del deporte hoy: Escenarios y desafíos. Revista Iberoamericana de Psicología del Ejercicio y el Deporte, 6(1), 144.

Jiménez. J., Rodríguez, J. y Castillo, E. (2001). Necesidad de formación psicopedagógica de los entrenadores deportivos. Ágora digital.

Judukis, J. (2007) Teorías implícitas sobre el aprendizaje y su relación con las prácticas pedagógicas en estudiantes de pedagogía en la Universidad de Magallanes y Profesores en ejercicio de la XII Región. Magallanes: Chile.

LLedo Figueres, E., Martínez Serrano, G., y Huertas Olmedo, F. (2014). Perfil del entrenador de fútbol en la etapa escolar en escuelas de clubes de élite de la Comunitat Valenciana (pp. 37-58). Murcia, España.

Lyle, J. (2007). Modelling the Complexity of the Coaching Process: A Commentary. International Journal of Sports Science and Coaching, 2(4), 408. 
Maestre, M., Garcés de los Fayos, E.J., Ortín, F.J., e Hidalgo, M.D. (2018). El perfil del entrenador excelente en fútbol base. Estudio mediante Grupos Focales. Cuadernos de Psicología del Deporte, 18(3), $112-128$

Manrique Arribas, J. C., Gea Fernández, J. M., \& Garzón, M. (2013). Perfil y expectativas del técnico de deporte escolar en el municipio de Segovia (España).

Marrero, J. (1993). Las teorías implícitas del profesorado. Vínculo entre la cultura y la práctica de la enseñanza. En M. J. Rodrigo, A. Rodríguez y J. Marrero: Las teorías implícitas. Una aproximación al conocimiento cotidiano. Madrid: Visor. Otras.

Monjas, R., Ponce, A. \& Gea, J.M. (2015). La transmisión de valores a través del deporte. Deporte escolar y deporte federado: relaciones, puentes y posibles trasferencias. Retos: Nuevas Tendencias en Educación Física, Deporte y Recreación, 28, 276- 284.

Mosston, M., \& Ashworth, S. (1986). Teaching physical education.

Mosston, M., \& Ashworth, S. (2008). Teaching physical education. First online edition.

Noguera, M. A. D. (1991). Los estilos de enseñanza en la Educación Física: Propuesta para una reforma de la enseñanza. Universidad de Granada. Instituto de Ciencias de la Educación.

Nuviala, A., León, J.A., Gálvez, J. \& Fernández, A. (2007). Qué actividades deportivas escolares queremos. Qué técnicos tenemos. Revista Internacional de Medicina y Ciencias de la Actividad Física y el Deporte, 25, 1-9.
Onofre, C. J. (1998). Didáctica de la Educación Física. Un enfoque constructivista. INDE.

Patiño, S., (2012). La enseñanza para la comprensión (EPC): propuesta metodológica centrada en el aprendizaje del estudiante. Revista Humanizarte Año 5, (8), pp.2-9.

Pedraza F., Durán V., Serna E., Hernández A., (2015). Los estilos de aprendizaje elemento clave en la interacción formativa: Caso licenciatura en deporte UPN. Revista Ímpetus, vol. 9 (2), pp. 91101.

Pérez-Muñoz, S., Chamorro-Juanes, P., Rodríguez-Cayetano, A., SánchezMuñoz, A., \& De Mena-Ramos, J. M. (2020). Efecto del modelo de enseñanza sobre la técnica individual de jugadores de fútbol sub-10. SPORT TK-Revista EuroAmericana de Ciencias del Deporte, 75-83.

Robles, M. T. A., Fuentes-Guerra, F. J. G., Rodríguez, J. R., \& López, J. M. R. (2011). Perfil, experiencia y métodos de enseñanza de los entrenadores de jóvenes futbolistas en la provincia de Huelva. Retos: nuevas tendencias en educación física, deporte y recreación, (20), 21-25.

Rodríguez, J., (2016). La influencia del estilo de enseñanza en el fútbol base. Sevilla España, pp.46 - 54.

Rodríguez, L. A. M., \& Quilindo, V. H. (2021). Enseñanza del fútbol e iniciación deportiva en dos escuelas deportivas de Santiago de Cali, Colombia. VIREF Revista de Educación Física, 10(1), 39-50. 
Schumacher, N., Schmidt, M., Wellmann, K., y Braumann, K. M. (2018). General perceptual-cognitive abilities: Age and position in soccer. Plos one, 13(8).

Valenzuela, A. V. (2005). Análisis de los cambios producidos en la metodología de la iniciación deportiva Descargar. Apunts. Educación física y deportes, 1(79), 59-67.

Zuluaga, Y. E. L., Isaza-Gómez, G. D., \& Osorio-Roa, D. M. (2021). Mediaciones educativas del entrenador en tiempo de crisis por COVID-19. Las características de esta publicación son propiedad de la Universidad Autónoma de Baja California., 170.

Zurita Ortega, F., Pérez Cortés, A. J., González Campos, G., Castro Sánchez, M., Chacón Cuberos, R., \&Ambris Sandoval, J. (2016). Estilos de enseñanza-entrenamiento y relación con ansiedad de jugadores de iniciación al futbol. Sportis, 2(3), 390-411. 